\title{
Inter-organ proteomic analysis reveals insights into the molecular mechanisms underlying the anti-diabetic effects of cis-9, trans-11 conjugated linoleic acid in ob/ob mice
}

\author{
V. Rungapamestry ${ }^{1}$, B. De Roos ${ }^{1}$, J. McMonagle ${ }^{2}$, G. Rucklidge ${ }^{1}$, M. Reid ${ }^{1}$, G. Duncan ${ }^{1}$, G. Horgan ${ }^{3}$, \\ A. P. Moloney ${ }^{4}$, S. Toomey ${ }^{2}$ and H. M. Roche ${ }^{2}$ \\ ${ }^{1}$ University of Aberdeen, Rowett Institute of Nutrition and Health, Aberdeen AB21 9SB, UK, ${ }^{2}$ Nutrigenomics Research \\ Group, UCD Conway Institute, University College Dublin, Dublin 4, Republic of Ireland, ${ }^{3}$ Biomathematics and \\ Statistics Scotland, Rowett Institute of Nutrition and Health, Aberdeen AB21 9SB, UK and ${ }^{4}$ Teagasc, Co. Meath, \\ Republic of Ireland
}

The major stereo-isomer of conjugated linoleic acid (CLA) in meat, cis-9 trans-11 CLA, exerts potent anti-diabetic effects in animal models of insulin resistance, such as the ob/ob mice, by improving insulin and lipid metabolism and decreasing obesity-induced inflammation $^{(1)}$. The aim was to further explore the effects of CLA on molecular biomarkers of the metabolic syndrome using a proteomic approach.

Male ob/ob mice (eight per group) received a cis-9 trans-11 CLA-rich diet which comprised either a high beef-derived CLA or a synthetic CLA diet, or a control diet for $28 \mathrm{~d}$. Diets were substituted with $36 \%(\mathrm{w} / \mathrm{w})$ either high- or low-CLA freeze-dried beef and comprised $52 \%$ energy derived from fat. Both CLA-rich diets contained $0.6 \%$ (w/w) cis- 9 trans-11 CLA. Fasting plasma biomarkers of insulin sensitivity were measured and changes in protein expression in skeletal muscle, hepatic and epididymal adipose tissues were determined using two-dimensional gel electrophoresis and $\mathrm{MS}^{(2)}$.

The CLA-fed mice were equally protected from the metabolic syndrome compared with the control, as shown by significant decreases of 30-50\% in plasma insulin $(P<0.05)$, glucose $(P<0.05)$, NEFA $(P<0.05)$, TAG $(P<0.05)$ and IL-6 $(P<0.05)$ and an increase of $34 \%$ in plasma adiponectin $(P<0.05)$. Proteomic analysis indicated that 134 , ninety-two and forty-three proteins, including post-translationally modified isoforms, were significantly altered $(P<0.05)$ in the skeletal muscle, hepatic and adipose tissues respectively of CLA-fed mice. The majority of these proteins were involved in cellular and oxidative stress, cytoskeletal integrity, glucose and lipid metabolism, membrane signalling and vesicular transport.

Decreased glyceroneogenesis and increased $\beta$-oxidation in the adipose tissue of CLA-fed mice may partly underlie their improved metabolic profile. Unexpectedly, key gluconeogenic enzymes were increased alongside an increased flux through the tricarboxylic acid cycle in their liver, which may indicate a complex coordination between hepatic gluconeogenesis and energy metabolism. Moreover, multiple positional variants of key regulatory glycogenolytic enzymes were up regulated in their skeletal muscle, with a concurrent decrease in $\beta$-oxidation. A host of cytoskeletal proteins were differentially modulated across all tissues and may reflect altered cytoskeletal re-organisation in these mice.

The biochemical interplay between these proteins will be further studied, using systems biology tools, to elucidate the molecular changes in proteomic signatures that may explain the systemic anti-diabetic effects observed following supplementation with CLA.

This work was supported by the Department of Agriculture and Food, Food Institutional Research Measure (FIRM) and by the Scottish Government Rural and Environment Research and Analysis Directorate (RERAD).

1. Moloney F, Toomey S, Noone E et al. (2007) Diabetes 56, 574-582.

2. de Roos B, Duivenvoorden I, Rucklidge G et al. (2005) FASEB J 19, 813-815. 